\title{
Carbon Protective Layer of Metal Evaporated Tape for Digital VCR
}

\author{
Hiroshi SEKI ${ }^{1}$, Hideyuki UEDA ${ }^{1}$, Kenji KUWAHARA ${ }^{1}$, Masaru ODAGIRI ${ }^{1}$ and Toshiaki KUNIEDA ${ }^{2}$ \\ 1) Materials and Devices Lab., Matsushita Electric Industrial Co., Ltd., \\ 1-4, Matsuo-cho, Kadoma, Osaka 571, JAPAN \\ 2) AV Device Technology Center, Matsushita Electric Industrial Co., Ltd., \\ 2-15, Matsuba-cho, Kadoma, Osaka 571, JAPAN
}

\begin{abstract}
The lubricity, durability and anti-conosive properties of metal evaporated tape (ME tape) for digital VCR were investigated. To improve the reliability of ME tape, hard Diamond-Like Carbon (DLC) film is applied as a protective layer on a Co-O magnetic layer. The $10 \mathrm{~nm}$ DLC film with a smooth and uniform morphology is prepared by a Plasma CVD method using methane and Ar gas. ME tape with DLC shows a stable frictional coefficient against a stainless steel pin. It seems that hard DLC film prevents an increase of the real contact area between ME tape and a stainless steel pin. Hard DLC film also improves still-frame life. The Ms fluctuation of the magnetic layer as a function of the storage period in $60^{\circ} \mathrm{C} 90 \%$ R.H. atmosphere was also investigated. DLC film is one of the most important factors to achieve an excellent anti-corrosive property with ME tape.
\end{abstract}

\section{I .INTRODUCTION}

Amazing progress has been made in digital recording media and Hi-definition media such as DCC, MD, HDTV, ATV, digital VCR and so forth. Metal evaporated tape (ME tape) is one of the most promising materials for high-density magnetic recording and superior CNR [1,2]. However the durability of the tape has been one of our concerns for improvement in practical use. We have developed ME tape for digital VCR or Hi-Definition digital VCR. Excellent reliability is achieved by controlling asperities distribution, applying hard Diamond-like Carbon (DLC) as a protective layer and applying fluorine-containing lubricant on DLC. Asperities distribution control is important to obtain a stable reproduced signal level for long period use [3]. It is necessary to distribute fine asperities which have a height of about $20 \mathrm{~nm}$ when a hard protective layer is applied. Big asperities generate uneven wear of magnetic heads near the recording gap, and this uneven wear causes spacing loss between the recording gap and the magnetic layer. Then the reproduced signal level will decrease in the long run. On the other hand, fluorine-containing lubricant reduces the frictional coefficient and improves water repellency of ME tape [4]. In this study, the durability of ME tape in both running and still-frame mode were investigated. The anti-corrosive properties were also investigated. The effects of the DLC protective layer on the reliability of ME tape are mainly discussed.

\section{II .EXPERIMENTAL}

Figure 1 shows a schematic cross sectional view of an example of the ME tape. Polyethylene terephthalate (PET) film was used as a non-magnetic substrate. An under coat layer was formed to distribute fine asperities which had a height of $20 \mathrm{~nm}$ with a density of $10^{5}$ to $10^{\circ}$ asperities $/ \mathrm{mm}^{2}$ according to AFM analysis. Co-O ferromagnetic metal thin film was deposited by an oblique vapor deposition method while applying oxygen gas. On the Co-O layer, DLC film was applied as a protective layer. $10 \mathrm{~nm}$ DLC film was prepared by a plasma CVD method using methane and Ar gas. Finally, a lubricant layer containing fluorine was formed on DLC film.

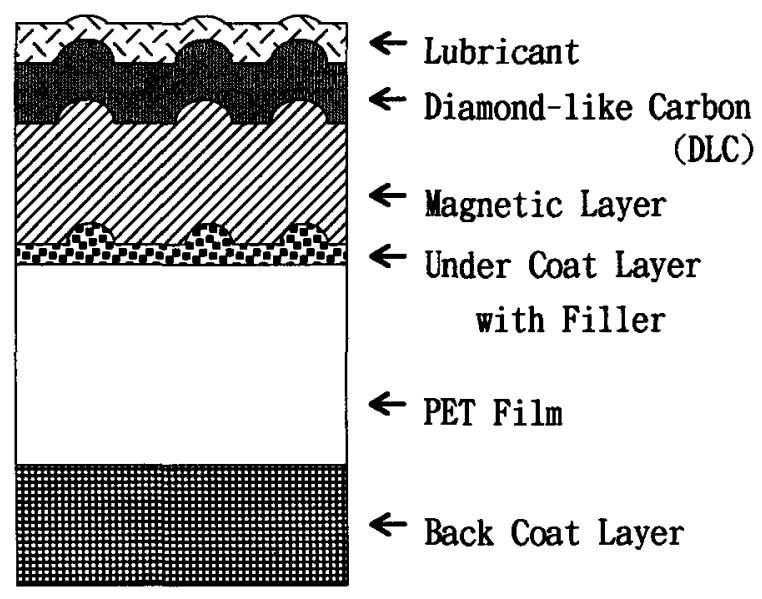

Fig. 1 Cross Section of ME Tape

Top and cross sectional view of the DLC protective layer were observed by SEM. The structure of the DLC was analyzed with Raman spectroscopy. The hardness of the 10 microns DLC on the silicon substrate was measured by Vickers hardness tester. 


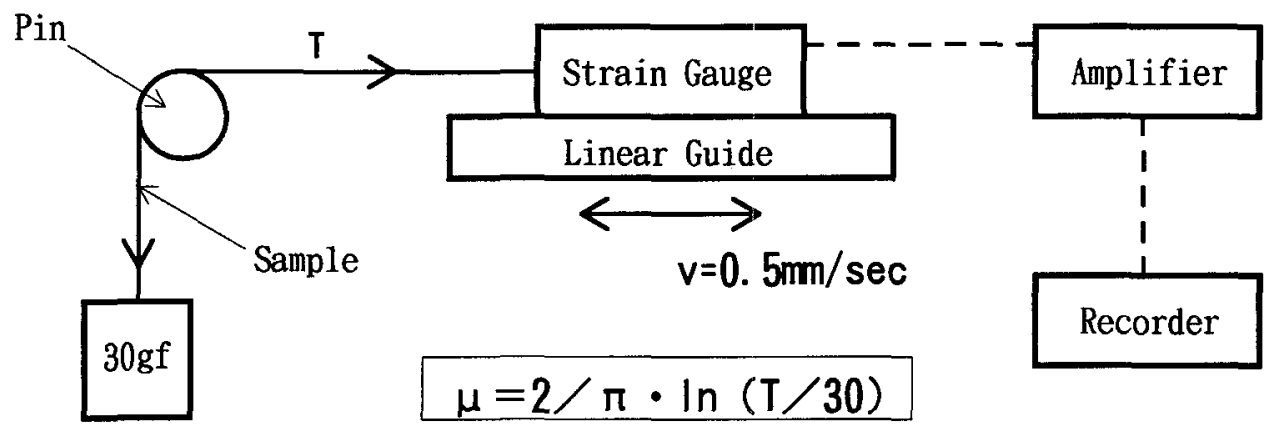

Fig. 2 Schematic Diagram of Friction Measurement Apparatus

The effects of the DLC protective layer on lubricity, durability and the anti-corrosive properties of ME tape were investigated. As samples, ME tape with DLC film and ME tape without DLC film were prepared for lubricity investigation. For durability and anti-corrosive property measurement, ME tape with an amorphous carbon (a-C) protective layer was added as an extra sample. The a-C film was formed by sputtering a graphite target with Ar gas. Frictional coefficients against a stainless steel pin with a diameter of $4 \mathrm{~mm}$ and a surface roughness $R \max =200 \mathrm{~nm}$ were measured by an apparatus as shown schematically in Figure 2. For these measurements, ME tapes with three different Rmax were prepared. Measurements were obtained by moving a sample tape forward and reverse against a steel pin for 25 cycles at $23{ }^{\circ} \mathrm{C}$ in $70 \%$ R.H. atmosphere. After applying a tension of $30 \mathrm{gf}$ to one end of the tape, the other end was moved at the rate of $0.5 \mathrm{~mm} / \mathrm{sec}$. Still-frame lives with the tape tension of 20 gf were investigated using existing $\mathrm{Hi}-8$ VCR in $23{ }^{\circ} \mathrm{C} 10 \%$ R.H. atmosphere. Still-frame life was defined as the time until there was a $50 \%$ signal reduction in the amplitude of the reproduced signal in still-frame mode. The anti-corrosive property, Ms (saturated magnetization) fluctuation of the magnetic layer of these samples was investigated as a function of

(a)

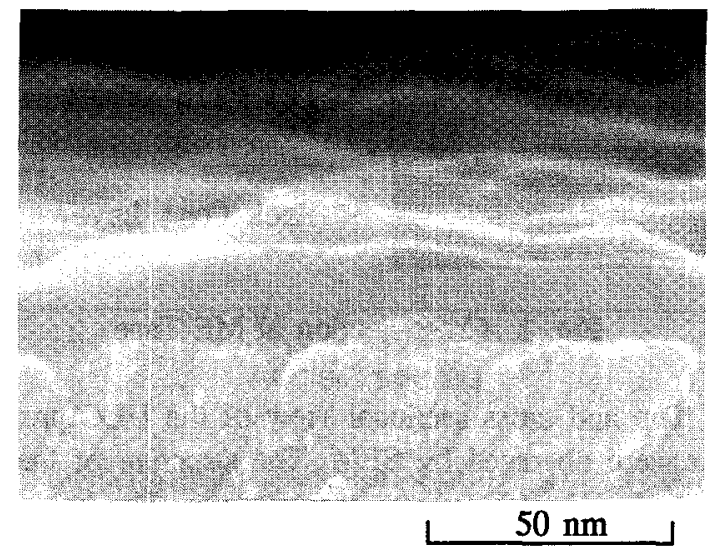

Fig. 3 SEM Micrographs of ME Tape the exposure period in $60{ }^{\circ} \mathrm{C} 90 \%$ R.H. atmosphere. As a severe and accelerated test, samples were placed on glass plates in a poremeric box and were exposed to $60^{\circ} \mathrm{C}$ 90\% R.H. atmosphere. To exclude any lubricant effect on the anti-corrosive properties, unlubricated samples were tested. Magnetic properties were measured by VSM.

\section{III.RESULTS AND DISCUSSION}

\section{A. SEM Observation}

SEM micrographs of the cross section and the surface of ME tape are shown in Figure 3. They indicate that DLC film has a very smooth and uniform morphology. The coverage property is also fine.

\section{B. Raman Spectroscopy and Hardness Measurement}

Figure 4 shows the Raman spectrum of carbon film prepared by the plasma CVD method using methane and Ar gas in this study. It was similar to a typical spectrum of Diamond-like Carbon [5]. The Vickers hardness of the DLC film was approximately $2500 \mathrm{~kg} / \mathrm{mm}^{2}$. On the other hand, the hardness of sputtered a-C film was $650 \mathrm{~kg} / \mathrm{mm}$ ${ }^{2}$. These results indicate that this chemical vapor deposited carbon film is very hard because it contains enough $\mathrm{sp}^{3}$ components in itself.

(b)

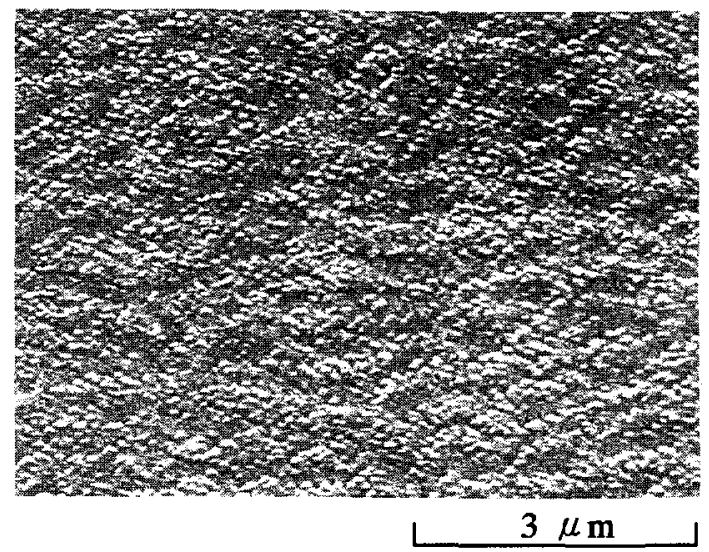
(a) ; Cross Section
(b) ; Top View 


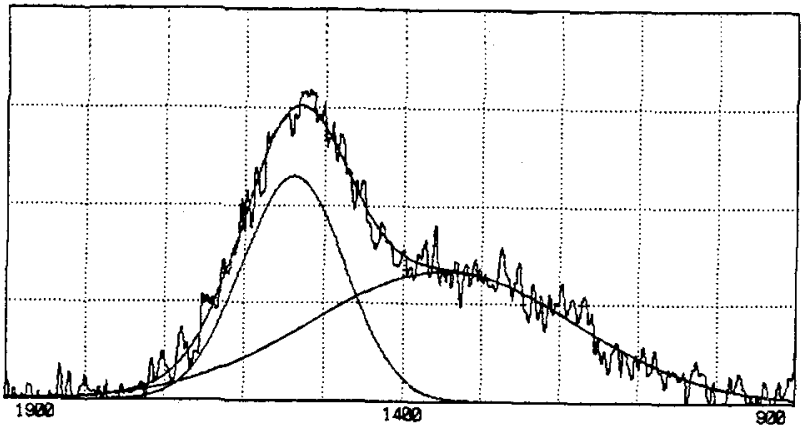

Fig. 4 Raman Spectrum of DLC Protective Layer

\section{Frictional Coefficient Measurement}

Figure 5 shows the difference in the frictional coefficient between lubricated ME tape with and without DLC. The horizontal axis of the graph indicates the surface roughness Rmax of the tape measured by AFM. The initial frictional coefficient of DLC deposited ME tape (symbol $O$ ) is better than that of ME tape without a DLC protective layer (symbol $\square$ ). After 25 passes, the frictional coefficient of ME tape without DLC increased (symbol $\square$ ), whereas DLC deposited tape indicated stable lubricity (symbol O). Unlubricated tape shows almost the same frictional coefficient at the rate of $0.5 \mathrm{~mm} / \mathrm{sec}$ as lubricated tape.

The difference in the initial frictional coefficient is explained below. As mentioned before, both lubricated and unlubricated tape show the same frictional coefficient. Therefore the interaction between the lubricant and DLC or the magnetic layer may be negligible in this case. In the case of ME tape without DLC, the magnetic layer and the stainless steel pin often are in direct contact, and adhesive friction and wear occur at the interface between them. On the other hand, DLC film has an inactive character, so that ME tape with DLC film does not show adhesive behavior. The increase in the frictional coefficient during the measurement is explained below. When there is no hard DLC protective layer, adhesive wear can easily occur at the interface. Wear of the magnetic layer means an increase in real contact area, which leads to an increase in frictional coefficient. Figure 6 shows surface roughness change in the sample without a carbon protective layer after the measurement. Hard DLC deposited ME tape does not show this kind of surface roughness change, which is caused by direct contact between the magnetic layer and the stainless steel pin.

These estimations indicate that the DLC protective layer plays two important roles. One is preventing adhesive friction between the magnetic layer and metal, and another is preventing any increase in the real contact area at the interface during tape slide.

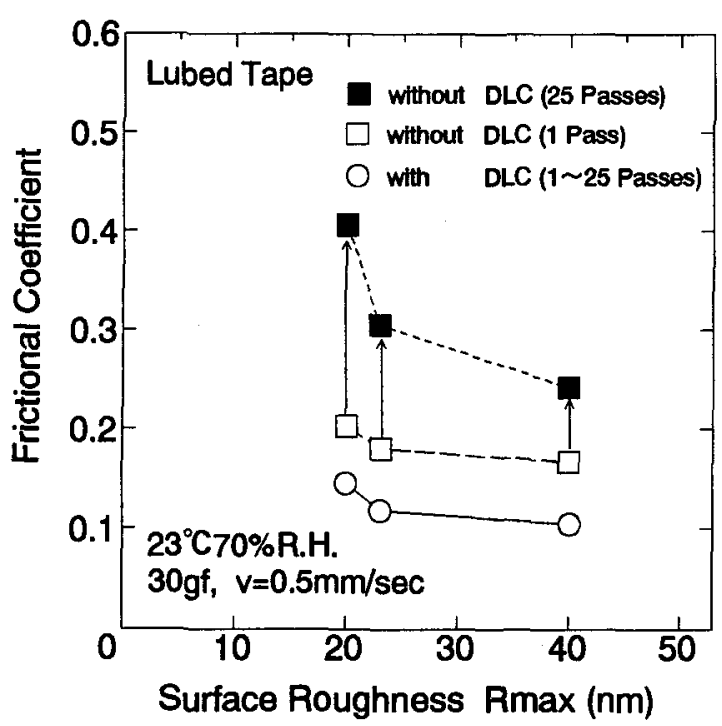

Fig. 5 Surface Roughness VS Frictional Coefficient
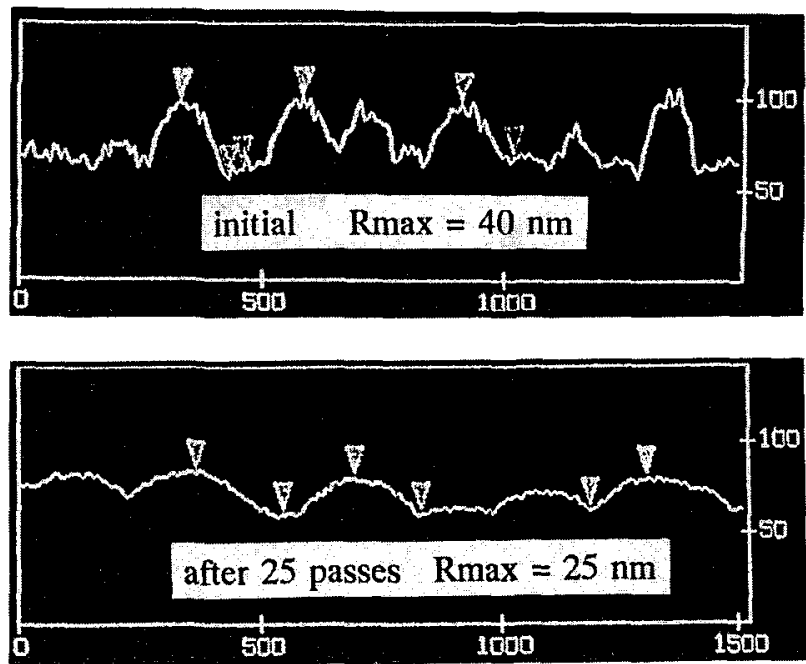

Fig. 6 Surface Roughness Change of ME Tape without DLC after Friction Measurement

\section{Still-Frame Life Measurement}

Figure 7 shows the still-frame life of DLC deposited ME tape, a-C deposited ME tape and ME tape without a carbon protective layer in $23{ }^{\circ} \mathrm{C} 10 \%$ R.H. atmosphere. If DLC film is not applied as a protective layer, contact and slide of head/tape in high speed mode causes wear of the tape. And Co debris which sticks to the surface of magnetic head can often be observed. A hard DLC protective layer improves durability in stop motion mode marvelously compared with a-Carbon. An inactive character and the hardness of the protective layer are important factors to improve still-frame life. 


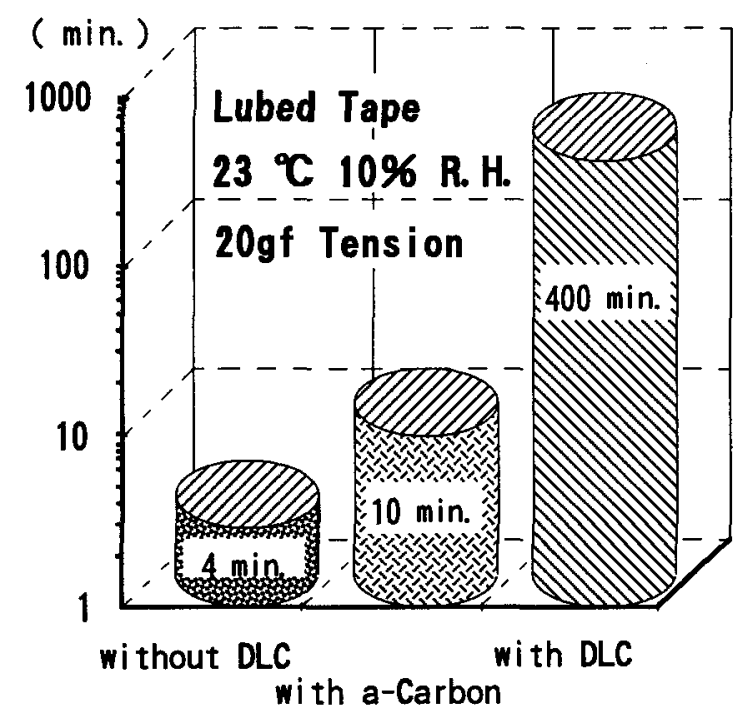

Fig. 7 Still-Frame Life of Three ME Tapes

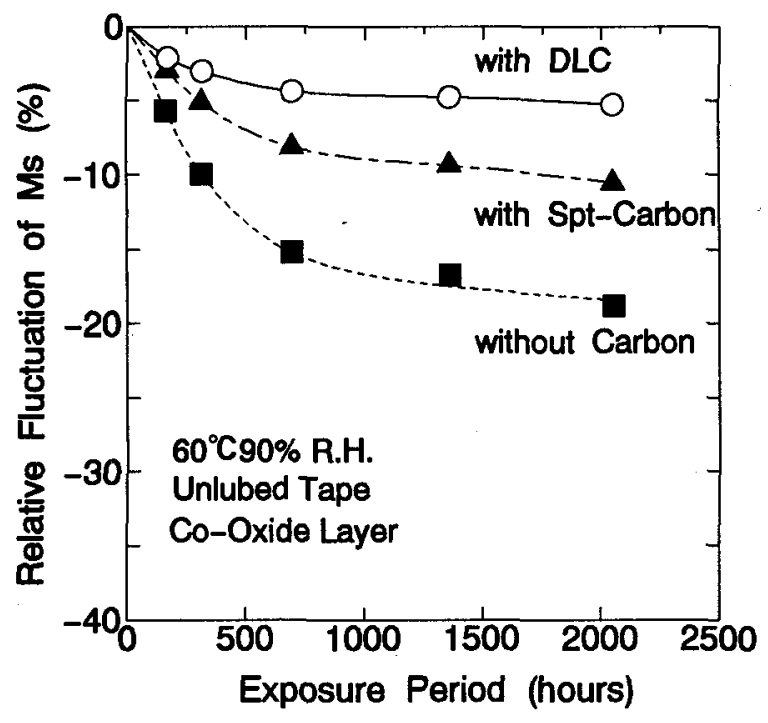

Fig. 8 Ms Fluctuation after $60{ }^{\circ} \mathrm{C}$ 90\% R.H. Exposure

\section{E. Anti-Corrosive Properties Measurement}

Figure 8 shows Ms fluctuation of the magnetic layer of the unlubricated samples as a function of the exposure period in $60{ }^{\circ} \mathrm{C} 90 \%$ R.H. atmosphere. Ms fluctuation of ME tape without a protective layer is larger than that of other tapes. On the other hand, DLC deposited tape has better anti-corrosive properties than carbon-sputtered tape. It is known that Ms fluctuation is caused by a composition change of $\mathrm{CoO}$ to $\mathrm{Co}(\mathrm{OH})_{2}$ or $\mathrm{CoOOH}$ at the surface of the magnetic layer [6]. The trigger of this composition change is $\mathrm{H}_{2} \mathrm{O}$ which mainly penetrates through the protective layer. By SEM observation, sputtered carbon film seems to be composed of fine clusters. Therefore the porosity of sputtered carbon film seems to be higher than that of the DLC film deposited by the CVD method. Pore-free DLC is one of the most important factors to achieve excellent anti-corrosive properties with ME tape. Of course, fluorine-containing lubricant increases the water repellency of ME tape, so that lubricated ME tape can achieve better anti-corrosive properties in practical use.

\section{IV.CONCLUSIONS}

The structure of a DLC protective layer prepared by a plasma CVD method were investigated by Raman spectroscopy and SEM observation. The performance of the DLC protective layer against the reliability, lubricity, durability and anti-corrosive properties of ME tape, were investigated, too. Excellent performance with ME tape was achieved by applying hard DLC film as a protective layer. DLC film also maintains a good balance between excellent recording/reproducing properties and running durability. The results obtained are as follows.

(1) ME tape with a DLC protective layer shows a low and stable frictional coefficient against a steel pin.

(2) The hard DLC protective layer improves durability in still-frame mode compared with sputtered carbon film.

(3) Ms fluctuation of DLC deposited ME tape in $60{ }^{\circ} \mathrm{C}$

90\% R.H. atmosphere is better than that of ME tape with a sputtered carbon protective layer and ME tape without a protective layer.

As mentioned above, the inactive character and hardness of the DLC protective layer improves the reliability of ME tape. In addition, applying a lubricant containing fluorine on the DLC protective layer is very effective to achieve more stable and excellent properties, specifically, reducing the frictional coefficient, improving the anti-wear property during high speed contact/slide between tape and head, and increasing the water repellency of ME tape.

\section{REFERENCES}

[1] B. Bhushan, Tribology and Mechanics of Magnetic Storage Devices, Springer-Verlag, New York, 1990

[2] C. D. Mee and E. D. Daniel, Magnetic Recording, McGraw-Hill, New York, 1987

[3] M. Murai, M. Odagiri, K. Takahashi, H. Youda and K. Shinohara : Proc. JAST Conf. Tokyo. p23, May 1990

[4] A. Tomago, M. Murai and S. Enomoto : Tech. Report IEICE, OME87-59, p29 (1987)

[5] M. Yoshikawa, G. Katagiri, H. Ishida, A. Ishitani and T. Akamastu : J. Appl. Phys., 64, 6464 (1988)

[6] N. Nouchi, Y. Kai, Y. Maezawa, N. Nakamura and K. Shinohara : J. Mag. Soc. Jpn, Vol.14, 251 (1990) 